\title{
3 Research Square

\section{Factors affecting viral load suppression in people living with HIV in Nigeria: cross-sectional analysis from 2001 to 2021}

Silviu Tomescu ( $\sim$ Silviu.Tomescu@righttocare.org )

Right to Care

Thomas Crompton

Right to Care

Jonathan Adebayo

Right to Care

Francis Akpan

Right to Care

Dauda Sulaiman Dauda

The Palladium Group

Zola Allen

The Palladium Group

Evans Ondura

The Palladium Group

Constance Wose Kinge

Right to Care

Charles Chasela

Right to Care

Pedro Pisa

Right to Care

\section{Research Article}

Keywords: unsuppressed VL, MMD, Nigeria, PLHIV

Posted Date: January 27th, 2022

DOI: https://doi.org/10.21203/rs.3.rs-1276836/v1

License: (a) (i) This work is licensed under a Creative Commons Attribution 4.0 International License.

Read Full License 


\section{Abstract \\ Background}

Human immunodeficiency virus (HIV) is untransmissible when the viral load (VL) is undetectable, underscoring the importance of achieving VL suppression for HIV epidemic control. It is estimated that $78 \%$ of people living with HIV (PLHIV) on antiretroviral therapy (ART) in Nigeria are virally suppressed. The need to improve $V L$ suppression rates raises questions about the factors associated with an unsuppressed VL, which this study explored using a large cross-sectional database of clients who received ART over a period of 20 years.

\section{Methods}

Data from 517,012 clients with a documented VL were analyzed using univariate and adjusted multivariate logistic regression. The independent variables investigated were sex, age group, facility type, time on ART until last VL test, multi-month dispensing (MMD), and geopolitical zone. To verify model improvement by variable, we performed backward elimination for the logistic regression model.

\section{Results}

Sixty-six percent of the study population were females. The largest age groups were 35-44 and 25-34, accounting for $32 \%$ and $31 \%$, respectively. The greatest adjusted odds for an unsuppressed VL were associated with shorter duration MMD prescriptions of 1-2 months (adjusted odds ratio [AOR]=14.05) and 3 months ( $A O R=3.13)$. Males had $8 \%$ greater odds ( $A O R=1.08)$ of being unsuppressed. The age groups below the 45-59 age group ( $A O R=0.84$ ) had higher odds of having an unsuppressed $V L$, with the highest odds in the $0-14$ age group (AOR=1.87). Clients enrolled at tertiary and secondary level facilities had the greatest odds of having an unsuppressed VL. A shorter time on ART until the last VL (1-3 years [AOR=1.14]) was associated with a higher risk of an unsuppressed VL. Clients in the North-Central $(A O R=1.83)$ and North-East $(A O R=1.49)$ zones had the greatest odds of viral non-suppression.

\section{Conclusions}

Enabling the provision of $3+$ months of MMD to PLHIV and targeting younger age groups, tertiary and secondary health facilities, small and medium facilities, and the North-Central and North-East zones for interventions could lead to improvements in VL suppression in Nigeria. The independent factors associated with an unsuppressed VL can guide improvements in ART program development and VL suppression of PLHIV in Nigeria.

\section{Background}


In 2020, 37.7 million people were living with HIV (PLHIV) globally; currently, 27.5 million (73\%) have access to antiretroviral therapy (ART) [1]. In 2020, $66 \%$ of PLHIV were virally suppressed [1]. Nigeria, a country with one of the highest global HIV infection rates, was reported to have $78 \%$ of PLHIV on ART virally suppressed, against a target of $95 \%[1,2]$. The Nigeria National Guidelines for HIV Prevention, Treatment, and Care define virologic suppression as having a viral load (VL) below 1,000 HIV ribonucleic acid copies per $\mathrm{mL}$ of plasma [3]. Given that an undetectable VL makes HIV untransmissible, suppressing the VL of $95 \%$ of PLHIV on ART is key to achieving epidemic control $[4,5]$.

Globally, including in some Nigerian States, factors that were found to be predictors of viral suppression were age, sex, duration on ART [6-9], current ART regimen [10], and adherence to medications [11]. This study explored whether similar associations existed broadly in Nigeria using data from 16 States over a period of 20 years, contributing to the body of knowledge and allowing better targeted interventions to improve HIV programs, the VL of clients, and epidemic control in the country.

The objective of this study was to determine which factors were associated with an unsuppressed VL in Nigeria using a large cross-sectional database of clients who received ART. We explored several variables -sex, age group, duration on ART, multi-month dispensing (MMD), facility tier, and geopolitical zone-to identify which factors were associated with viral non-suppression. Results of this analysis can guide HIV programs to target population groups at the highest risk.

\section{Methods}

\section{Study design, setting, and population}

The study was a retrospective cross-sectional analysis of clients who were enrolled on ART at 580 facilities across 16 States in Nigeria that were supported by the United States Agency for International Development (USAID). The data set covered a period of 20 years, with the first ART initiation date recorded on January 1, 2001, and the most recent ART pickup date of March 26, 2021. The study was carried out to investigate the clinical and demographic factors associated with an unsuppressed VL among more than 500,000 HIV clients who had a VL test during the period.

\section{Data Source and Management}

The data were provided by the USAID-supported implementing partners (IPs) through their quarterly data submission using the retention and audit determination tool (RADET). Each IP submission was then combined into a single data set that was used for this study.

The data obtained were collected by the Federal Ministry of Health (FMOH) and IPs using standardized national HIV data collection tools that recorded demographic, clinical, and treatment information about the client at each visit in the electronic medical records system.

The data set received contained 867,981 non-longitudinal, cross-sectional client records. Due to missing unique client identifiers for 158,898 clients, a unique identifier was created for data deduplication using 
the date of birth, sex, database-provided unique identifier, and client hospital number. Data cleaning involved removing duplicate unique identifiers $(n=17,267)$, and missing entries for MMD $(n=1)$ and ART regimen line ( $n=148)$. In addition, records with an ART start date occurring before the date of birth or after the date of last drug pickup, or a VL result date occurring earlier than the ART start date, were removed from the sample $(n=3,030)$. Clients with a date of birth earlier than $1940(n=961)$ or an ART start date earlier than $1990(n=2)$ were also excluded from the analysis. An additional 2,314 clients with a VL test result date earlier than the ART start date were likewise removed. Missing values for current ART status $(n=56)$ and sex $(n=1)$ were excluded (Figure 1$)$.

Of the 844,201 complete client records retained, 517,012 (61.2\%) had a VL test (only the last one) on record, whereas the remaining 327,189 (38.8\%) had no documented VL test (Figure 1). Clients without documented $V L$ test results were removed from the study population before the logistic regression analysis was conducted.

\section{Variables explored as predictors of unsuppressed viral load}

The "age at last VL test" variable was generated by calculating the time difference (in years) between the date of received current $\mathrm{VL}$ and the date of birth of the client. The age at last $\mathrm{VL}$ test was reclassified into six age groups: $0-14,15-24,25-34,35-44,45-59$, and $60+$ years.

Similarly, the "duration on ART to last VL test" variable was created by calculating the time difference (in months) between the date of received current VL and the ART start date. The duration on ART was reclassified as $<1$ year, $2-3$ years, and $3+$ years, and labeled as "time to last VL."

The "facility size" variable was calculated by determining the number of clients ever receiving care at the facility, then assigning the facility size group as small $[0,25)$ percentiles, medium $[25,75)$ percentiles, or large $[75,100]$ percentiles.

The "facility tier" variable was created by classifying the facility name provided in the data set to the nationally recognized facility tiers using the Nigeria health facility registry (HFR) [12]. The classification resulted in three levels of facilities: primary, secondary, and tertiary. Similarly, the "facility ownership" variable was created by grouping the facilities in their respective ownership type (public or private) according to their classification in the Nigeria HFR system [12].

The "geopolitical zone" variable was created by grouping the 16 USAID-supported States into their nationally recognized geopolitical zones. This resulted in five geopolitical zone groupings: North-Central (Kwara and Niger States), North-East (Adamawa, Bauchi, Borno, and Yobe States), North-West (Jigawa, Kano, Kebbi, Sokoto, and Zamfara States), South-South (Akwa Ibom, Bayelsa, Cross River, and Edo States), and South-West (Lagos).

\section{Statistical analysis}


For the population with a last VL test on record, the Pearson's chi-square test was used to examine the association of each variable with an unsuppressed VL at a client's last VL test date. Unadjusted and adjusted logistic regression models were run to explore the association of variables with an unsuppressed VL. Current ART status was excluded as a factor from the regression modeling because at the time of the VL test, all clients were active, even though they may now have a different status (interruption in treatment [IIT], deceased, or stopped treatment). Similarly, the regimen line was excluded from the regression analysis because other regimen lines, aside from the first-line regimen, are prescribed in the case of an unsuppressed VL. The regimen line was therefore dependent on the VL outcome investigated and it could not be used as an independent variable associated with VL non-suppression. The variables included in the models were sex, age group, geopolitical zone, facility tier, facility size, MMD of antiretrovirals (ARVs), and time to last VL test. The group accounting for the most clients in each of the independent variables analyzed was set as the reference group for the respective variable. A two-tailed $P$ value of $P<0.000001$ was used to define statistical significance [13]. Model selection was done using backward elimination where the Akaike's information criterion (AIC) was used to evaluate variable inclusion in the final model. All data were analyzed using R software for Statistical Computing v4.0.5 [14].

\section{Results}

Of the 517,012 clients included in the analysis, 476,218 (92\%) were virologically suppressed whereas the remaining $8 \%$ were virologically unsuppressed (Table 1 ). Sixty-six percent of the clients were female, with $92 \%$ of both sexes virologically suppressed. Clients ages $25-34$ and 35-44 were the largest age groups, accounting for $31 \%$ and $32 \%$ of the total number of clients in the analysis, respectively. Clients in the $0-$ 14 age group had the smallest proportion who were virally suppressed. 
Table 1

Client characteristics, grouped by viral suppression

\begin{tabular}{|c|c|c|c|c|}
\hline Factors & Unsuppressed (\%) & Suppressed (\%) & Total (\%) & p-value \\
\hline \multicolumn{5}{|l|}{ Sex } \\
\hline Female & $26,558(8)$ & $312,401(92)$ & $338,959(66)$ & 0.042873 \\
\hline Male & $14,236(8)$ & $163,817(92)$ & $178,053(34)$ & \\
\hline \multicolumn{5}{|l|}{ Age group } \\
\hline $35-44$ & $12,045(7)$ & $152,752(93)$ & $164,797(32)$ & $<0.000001$ \\
\hline $25-34$ & $12,157(8)$ & $149,699(92)$ & $161,856(31)$ & \\
\hline $45-59$ & $7,006(7)$ & $98,026(93)$ & $105,032(20)$ & \\
\hline $15-24$ & $4,535(9)$ & $44,231(91)$ & $48,766(9)$ & \\
\hline $60+$ & $1,278(7)$ & $17,985(93)$ & $19,263(4)$ & \\
\hline $0-14$ & $3,773(22)$ & $13,525(78)$ & $17,298(3)$ & \\
\hline \multicolumn{5}{|l|}{ Current ART status } \\
\hline Active & $22,786(5)$ & $428,619(95)$ & $451,405(87)$ & $<0.000001$ \\
\hline IIT & $10,370(26)$ & $29,132(74)$ & $39,502(8)$ & \\
\hline Transferred out & $3,834(24)$ & $12,014(76)$ & $15,848(3)$ & \\
\hline Dead & $3,104(38)$ & $5,024(62)$ & $8,128(2)$ & \\
\hline Stopped & $700(33)$ & $1,429(67)$ & $2,129(0)$ & \\
\hline \multicolumn{5}{|c|}{ Current regimen line } \\
\hline Adult 1st line & $35,558(7)$ & $456,863(93)$ & $492,421(95)$ & $<0.000001$ \\
\hline Pediatric 1st line & $2,706(22)$ & 9,695 (78) & $12,401(2)$ & \\
\hline Adult 2nd line & $2,389(20)$ & $9,438(80)$ & $11,827(2)$ & \\
\hline Pediatric 2nd line & $123(40)$ & $182(60)$ & $305(0)$ & \\
\hline Adult 3rd line & $10(29)$ & $24(71)$ & $34(0)$ & \\
\hline Salvage & $8(33)$ & $16(67)$ & $24(0)$ & \\
\hline \multicolumn{5}{|l|}{ Facility tier } \\
\hline Secondary & $25,233(9)$ & $254,063(91)$ & $279,296(54)$ & $<0.000001$ \\
\hline Primary & $8,067(5)$ & $163,076(95)$ & $171,143(33)$ & \\
\hline Tertiary & 7,494 (11) & $59,079(89)$ & $66,573(13)$ & \\
\hline
\end{tabular}




\begin{tabular}{|c|c|c|c|c|}
\hline Factors & Unsuppressed (\%) & Suppressed (\%) & Total (\%) & p-value \\
\hline \multicolumn{5}{|c|}{ Facility Ownership } \\
\hline Public & $38,124(8)$ & $448,514(92)$ & $486,638(94)$ & $<0.000001$ \\
\hline Private & $2,670(9)$ & $27,704(91)$ & $30,374(6)$ & \\
\hline \multicolumn{5}{|l|}{ Facility size } \\
\hline Large & $32,055(7)$ & $400,824(93)$ & $432,879(84)$ & $<0.000001$ \\
\hline Medium & $8,337(11)$ & $70,774(89)$ & $79,111(15)$ & \\
\hline Small & 402 (8) & $4,620(92)$ & $5,022(1)$ & \\
\hline \multicolumn{5}{|l|}{ MMD } \\
\hline $3+$ & $10,419(3)$ & 308,307 (97) & $318,726(62)$ & $<0.000001$ \\
\hline 3 & $15,094(10)$ & $135,703(90)$ & $150,797(29)$ & \\
\hline $1-2$ & $15,281(32)$ & $32,208(68)$ & $47,489(9)$ & \\
\hline \multicolumn{5}{|l|}{ Time to last VL } \\
\hline $3+$ years & $18,994(9)$ & $200,513(91)$ & $219,507(42)$ & $<0.000001$ \\
\hline$<1$ year & $11,031(6)$ & $166,782(94)$ & $177,813(34)$ & \\
\hline $1-3$ year & $10,769(9)$ & $108,923(91)$ & $119,692(23)$ & \\
\hline \multicolumn{5}{|l|}{ Zone } \\
\hline South-South & $16,985(6)$ & $264,688(94)$ & $281,673(54)$ & $<0.000001$ \\
\hline North-East & $8,137(10)$ & $71,155(90)$ & $79,292(15)$ & \\
\hline North-West & $6,065(10)$ & $57,112(90)$ & $63,177(12)$ & \\
\hline South-West & $4,981(9)$ & $49,052(91)$ & $54,033(10)$ & \\
\hline North-Central & $4,626(12)$ & $34,211(88)$ & $38,837(8)$ & \\
\hline
\end{tabular}

The ART status of $87 \%$ of the clients was recorded as active on treatment, with the remaining $13 \%$ being dead (2\%), had transferred out to another facility (3\%), or had interrupted treatment (8\%) (Table 1). Most clients (95\%) were on the adult first-line ART regimen, with $93 \%$ of them being virally suppressed. Both the adult second-line and pediatric first-line ART regimens had $2 \%$ of the clients, with $80 \%$ and $78 \%$ of clients on these regimens being virally suppressed, respectively.

Most clients were receiving ART at a secondary health facility (54\%), followed by primary health facilities (33\%) (Table 1). Clients receiving treatment at a tertiary or secondary health facility were more likely to be virally unsuppressed compared with clients receiving ART at a primary health facility. 
Ninety-four percent of the clients received ARVs from a publicly owned facility, with $92 \%$ of them being virally suppressed (Table 1 ). Only $6 \%$ of the clients were receiving treatment from a privately owned facility, with $91 \%$ of these clients virally suppressed.

A greater proportion of ART clients received treatment from a large volume facility (84\%), with $93 \%$ of these clients being virally suppressed (Table 1). ART clients at the medium volume facilities comprised $15 \%$ of the total clients, with $89 \%$ of them being virally suppressed.

MMD of more than three months (62\%) and three months (29\%) of ARVs were the majority (Table 1$)$. These two groups had the highest proportions of virally suppressed clients on treatment, at $97 \%$ and $90 \%$, respectively.

Forty-two percent of the clients had been on ART for more than three years and $34 \%$ had been on treatment for less than one year (Table 1). Clients who were on ART for less than one year had a higher proportion of being virally suppressed (94\%) than those who had been on ART for one to three years or more than three years (both 91\%).

The South-South zone had the largest proportion of clients (54\%) in the sample and had the highest proportion of virally suppressed clients (94\%) (Table 1 ).

A multivariate logistic regression model was run using all variables in Table 1. All variables were significant, except for "Facility Ownership" ( $p$-value $=0.64925$ ). The AIC for this model did not change when ownership was included $(A I C=243,815)$, therefore it was removed from the model. Backward elimination of variables produced the lowest AIC-243,815 with all variables combined-that is, sex, time to last VL, facility tier, facility size, age group, geopolitical zone, and MMD. The logistic regression model assessing the relationship between predictor variables and an unsuppressed VL among ART clients is summarized in Table 2. All factors used in the adjusted logistic regression model were statistically significant, with a p-value of $<0.000001$, except for the $<1$ year time to last VL, which had a p-value of 0.18893 , and small facility size, with a p-value of 0.00029 . 
Table 2

Factors associated with an unsuppressed viral load

\begin{tabular}{|c|c|c|c|c|}
\hline \multirow[t]{2}{*}{ Factors } & \multicolumn{2}{|l|}{ Unadjusted OR } & \multicolumn{2}{|l|}{ Adjusted OR } \\
\hline & OR [95\% Cl] & p-value & AOR [95\% Cl] & p-value \\
\hline \multicolumn{5}{|l|}{ Sex } \\
\hline Female & 1 [ref] & & 1 [ref] & \\
\hline Male & 1.02 [1.00-1.04] & 0.042321 & $1.08[1.05-1.1]$ & $<0.000001$ \\
\hline \multicolumn{5}{|l|}{ Age group } \\
\hline $35-44$ & 1 [ref] & & 1 [ref] & \\
\hline $0-14$ & 3.54 [3.40-3.68] & $<0.000001$ & $1.87[1.79-1.95]$ & $<0.000001$ \\
\hline $15-24$ & 1.30 [1.25-1.35] & $<0.000001$ & 1.4 [1.35-1.45] & $<0.000001$ \\
\hline $25-34$ & 1.03 [1.00-1.06] & 0.027536 & $1.1[1.07-1.13]$ & $<0.000001$ \\
\hline $45-59$ & $0.91[0.88-0.93]$ & $<0.000001$ & $0.83[0.81-0.86]$ & $<0.000001$ \\
\hline $60+$ & $0.90[0.85-0.96]$ & 0.000633 & $0.78[0.73-0.83]$ & $<0.000001$ \\
\hline \multicolumn{5}{|l|}{ Facility tier } \\
\hline Secondary & 1 [ref] & & 1 [ref] & \\
\hline Primary & $0.5[0.49-0.51]$ & $<0.000001$ & $0.74[0.72-0.76]$ & $<0.000001$ \\
\hline Tertiary & $1.28[1.24-1.31]$ & $<0.000001$ & $1.16[1.12-1.19]$ & $<0.000001$ \\
\hline \multicolumn{5}{|l|}{ Facility size } \\
\hline Large & 1 [ref] & & 1 [ref] & \\
\hline Medium & $1.47[1.44-1.51]$ & $<0.000001$ & $1.42[1.38-1.46]$ & $<0.000001$ \\
\hline Small & $1.09[0.98-1.21]$ & 0.106851 & $1.22[1.1-1.36]$ & 0.00029 \\
\hline \multicolumn{5}{|c|}{ Time to last VL } \\
\hline $3+$ years & 1 [ref] & & 1 [ref] & \\
\hline$<1$ year & $0.70[0.68-0.72]$ & $<0.000001$ & $0.98[0.95-1.01]$ & 0.18893 \\
\hline $1-3$ years & $1.04[1.02-1.07]$ & 0.000709 & $1.14[1.11-1.18]$ & $<0.000001$ \\
\hline \multicolumn{5}{|l|}{ MMD } \\
\hline $3+$ months & 1 [ref] & & 1 [ref] & \\
\hline 1-2 months & 14.04 [13.66-14.43] & $<0.000001$ & $14.05[13.64-14.48]$ & $<0.000001$ \\
\hline
\end{tabular}




\begin{tabular}{|lllll|}
\hline Factors & Unadjusted OR & & Adjusted OR & \\
\cline { 2 - 5 } & OR [95\% Cl] & p-value & AOR [95\% Cl] & p-value \\
\hline 3 months & $3.29[3.21-3.38]$ & $<0.000001$ & $3.13[3.05-3.22]$ & $<0.000001$ \\
\hline Zone & & & & \\
\hline South-South & $1[\mathrm{ref}]$ & & $1[$ ref] & \\
\hline North-Central & $2.11[2.04-2.18]$ & $<0.000001$ & $1.83[1.76-1.9]$ & $<0.000001$ \\
\hline North-East & $1.78[1.73-1.83]$ & $<0.000001$ & $1.49[1.45-1.54]$ & $<0.000001$ \\
\hline North-West & $1.65[1.60-1.71]$ & $<0.000001$ & $0.89[0.86-0.93]$ & $<0.000001$ \\
\hline South-West & $1.58[1.53-1.64]$ & $<0.000001$ & $0.74[0.72-0.77]$ & $<0.000001$ \\
\hline
\end{tabular}

Males (adjusted odds ratio $[\mathrm{AOR}]=1.08,95 \% \mathrm{Cl}$ : 1.05-1.10, $\mathrm{P}<0.000001)$ were found to have $8 \%$ higher odds of being virally unsuppressed than females (Table 2). Univariate and multivariate logistic regression indicated with statistical significance that young people ages 0-24 were associated with higher odds of viral non-suppression compared with the 35-44 age group. Younger clients ages $0-14$ years had the highest adjusted odds (AOR $=1.87,95 \% \mathrm{Cl}: 1.79-1.95, \mathrm{P}<0.000001)$.

Compared with the South-South zone, clients in the North-Central (AOR $=1.83,95 \% \mathrm{Cl}: 1.76-1.9)$ and North-East $(A O R=1.49,95 \% \mathrm{Cl}: 1.45-1.54)$ zones had greater odds of $\mathrm{VL}$ non-suppression $(\mathrm{P}<0.000001)$ (Table 2). Clients in the South-West zone had the lowest odds for VL non-suppression ( $A O R=0.74,95 \%$ Cl: 0.72-0.77).

Clients receiving ARVs at the tertiary health facilities were $16 \%$ more likely to be virally unsuppressed $(A O R=1.16,95 \% \mathrm{Cl}: 1.12-1.19)$ whereas primary health facilities were the most protected $(A O R=0.74$, $95 \% \mathrm{Cl}: 0.72-0.76)$ (Table 2). Moreover, small (AOR=1.22, 95\% Cl: $1.1-1.36, \mathrm{P}=0.00029)$ and medium $(A O R=1.42,95 \% \mathrm{Cl}: 1.38-1.46, \mathrm{P}<0.000001)$ facilities were found to have the highest odds for viral nonsuppression compared with large facilities.

The MMD was significantly associated with viral non-suppression. ART clients on 1-2 MMD had the highest odds of viral non-suppression rates ( $\mathrm{AOR}=14.05,95 \% \mathrm{Cl}$ : 13.64-14.48) in the adjusted logistic regression model.

Compared with clients who had been on ART for more than three years, those who had been on treatment between 1 and 3 years were 14\% more likely to be virally unsuppressed (AOR=1.14, 95\% Cl: 1.11-1.18), whereas clients on ART for less than one year were found to be $2 \%$ less likely to be virally unsuppressed (AOR=0.98, 95\% Cl: 0.98-1.01).

\section{Discussion}


Our study found that younger age groups that were not on MMD had the highest association with VL nonsuppression. We also found that males, clients who received treatment at tertiary health facilities, at small or medium facilities, and in the North-Central or North-East zones were associated with a higher risk of VL non-suppression. Other studies have found similar results for the risk of viral non-suppression in younger age groups in Cambodia, Uganda, and South Carolina (USA) $[6,8,15]$, and among males [1618].

The increased odds of viral non-suppression among ART clients who received treatment at tertiary health facilities were unlike the higher odds reported for primary health facilities in Ethiopia [19]. Small and medium facilities were identified as having greater odds for viral non-suppression; this was consistent with findings that clients were more likely to miss consecutive visits at lower volume facilities [20].

ART clients who had their last VL test conducted within less than 1 year on treatment were less likely to be virally unsuppressed compared with clients who had their VL tested conducted within 1 to 3 years of being on ART. Our finding was consistent with the Center for Disease Control and Prevention's finding that PLHIV on ART could be virally suppressed within six months of initiation, provided that they adhered to their medication [21]. Moreover, a greater likelihood of viral suppression was found among PLHIV who were initiated on treatment for less than one year compared with those on ART for more than one year [22]. However, contrary to this, a shorter time on ART was identified as a factor associated with an unsuppressed VL in South Africa [23]. The contradictory findings in the literature could speak to the specific adherence patterns of the populations investigated and models that may be specific to the study settings.

Short duration MMD of 1 to 3 months was identified as having the highest odds for an unsuppressed VL in both the univariate and covariate models. This is interesting because shorter MMD is also associated with non-adherence [24]. Shorter MMD is initially prescribed to new clients and longer MMD is prescribed to virally stable clients to reduce the number of clinic visits [25]. Because of non-adherence, clients may become virally unsuppressed and be placed on shorter duration treatment, requiring more frequent checkups and clinical support to become stable. It is apparent that MMD is dependent on the VL status of clients; however, it is not the case for new clients. The logistic regression model we built suggested that a shorter MMD was associated with an unsuppressed VL. In this case, the MMD was a confounding factor that possibly represented some degree of IIT because it was not the actual length of the prescription that can affect the $V L$, rather its misadministration. We found value in retaining the confounding factor in the analysis because we cannot use a factor like IIT instead, because, at the time of the VL test, the client was active on treatment. In the absence of longitudinal data that can identify IIT as a precedent to unsuppressed VL, we chose to include MMD in the analysis as a proxy indicator. In doing so, the recommendation of activating 3+MMD to support viral suppression is still valid, even if the improvement in VL suppression is caused by correct adherence to treatment, not by the actual length of MMD. The inclusion of MMD as an independent variable is a limitation of the study. 
A possible explanation for the North-Central zone having the highest odds of viral non-suppression could be linked to health-seeking behavior, poor adherence to treatment, and possibly religious beliefs $[26,27]$.

One of the major limitations of our study was the inaccessibility of the longitudinal data set, leaving us unable to conduct a longitudinal study to explore the factors affecting viral non-suppression over time. We were therefore restricted to conducting a cross-sectional study. Other unavailable variables that could be explored in future studies to identify their association with viral suppression are tuberculosis status, adherence level, ART drug regimen, marital status, and education level. The absence of unique client identifiers and VL suppression data for adolescents and recently initiated clients may have also had an impact on the study, suggesting that these findings may not necessarily apply to these sub population groups.

\section{Conclusions}

Enabling the provision of $3+$ months of MMD to PLHIV and targeting younger age groups (below 35), tertiary health facility, small and medium facilities, and the North-Central and North-East geopolitical zones for interventions could lead to improvements in VL suppression in Nigeria. The independent factors associated here with an unsuppressed VL can guide the improvement of ART program development and VL suppression of PLHIV in Nigeria.

\section{Abbreviations}

AIC

Akaike information criterion

AOR

Adjusted odds ratio

ART

Antiretroviral therapy

ARV

Antiretroviral

Cl

Confidence interval

\section{Data.FI}

Data for Implementation

FMOH

Federal Ministry of health

HFR

Health facility registry

HIV

Human immunodeficiency virus

IIT 
Interruption in treatment

IP

Implementing partner

MMD

Multi-month dispensing

PEPFAR

United States President's Emergency Plan for AIDS Relief

PLHIV

People living with HIV

RADET

Retention audit determination tool

\section{UNAIDS}

Joint United Nations Programme on HIV/AIDS

USAID

United States Agency for International Development

VL

Viral load

\section{Declarations}

\section{Ethical approval and consent to participate}

Ethical approvals for this study were obtained in Nigeria and the United States. Informed consent was waived from all subjects or, if subjects are under 18, from a parent and/or legal guardian by the expedited institutional review board (IRB) approvals granted by both the National Health Research Ethics Committee of Nigeria (NHREC), reference number NHREC/01/01/2007, and the HML IRB in the United States, reference number 772EQH20. Data were anonymised and handled confidentially during all phases of the research. All methods were carried out in accordance with relevant guidelines and regulations. All experimental protocols were granted approval by the institutional

review board (IRB) of the National Health Research Ethics Committee of Nigeria (NHREC), reference number NHREC/01/01/2007, and the HML IRB in the United States, reference number 772EQH20.

\section{Availability of data and materials}

The data that support the findings of this study are owned by the Government of Nigeria and were used under license for the current study. Access to these data is subject to restrictions owing to privacy and ethics policies set by the Government of Nigeria so are not publicly available. Requests to access these data should be directed to Dauda.Sulaiman@thepalladiumgroup.com.

\section{Competing interests}


The authors have declared that no competing interests exist.

Funding - This study has been made possible by the generous support of the American people and the United States President's Emergency Plan for AIDS Relief (PEPFAR) through USAID, including bilateral support through USAID Nigeria's Data for Implementation (Data.FI) mechanism under the terms of Cooperative Agreement 7200AA19CA0004 to Palladium and Right to Care. The contents are the responsibility of the authors and do not necessarily reflect the views of PEPFAR, USAID, or the United States Government. The funders had no role in the study design, data collection and analysis, decision to publish, or preparation of the manuscript. Right to Care, South Africa covers the salaries of ST, TC, JA, FA, CWK, CC and PP. The Palladium Group, Nigeria, covers the salaries of DSD, ZA and EO.

\section{Authors' contributions}

Study Design: ST, TC, JA, FA, CC, CWK, PP; Data collection: JA, DSD, EO; Data analysis: ST, TC, JA; Funding acquisition: DSD, FA, PP; Data Interpretation: All authors; Writing - original draft: ST, TC, JA; Writing - review and editing: All authors. All authors read and approved the final manuscript.

\section{Acknowledgements}

The authors acknowledge the role of USAID/Nigeria and PEPFAR implementing partners in supporting the Federal Ministry of Health of Nigeria $(\mathrm{FMOH})$ with data collection, cleaning, management and providing us with the opportunity to conduct this analysis.

\section{References}

1. Joint United Nations Programme on HIV/AIDS (UNAIDS). Global HIV \& AIDS statistics - Fact sheet. 2021. https://www.unaids.org/en/resources/fact-sheet. Accessed 28 Sep 2021.

2. UNAIDS. The gap report. 2014.

https://www.unaids.org/sites/default/files/media_asset/UNAIDS_Gap_report_en.pdf. Accessed 13 Sep 2021.

3. Federal Ministry of Health. National guidelines for HIV prevention, treatment and care. 2020.

4. Eisinger RW, Dieffenbach CW, Fauci AS. HIV viral load and transmissibility of HIV infection: Undetectable equals untransmittable. JAMA. 2019;321(5):451-2.

5. Montaner JSG, Hogg R, Wood E, Kerr T, Tyndall M, Levy AR, et al. The case for expanding access to highly active antiretroviral therapy to curb the growth of the HIV epidemic. The Lancet. 2006;368(9534):531-6.

6. Chhim K, Mburu G, Tuot S, Sopha R, Khol V, Chhoun P, et al. Factors associated with viral nonsuppression among adolescents living with HIV in Cambodia: a cross-sectional study. AIDS Res Ther. 2018;15(1):20.

7. Lokpo SY, Ofori-Attah PJ, Ameke LS, Obirikorang C, Orish VN, Kpene GE, et al. Viral Suppression and Its Associated Factors in HIV Patients on Highly Active Antiretroviral Therapy (HAART): A 
Retrospective Study in the Ho Municipality, Ghana. AIDS Res Treat. 2020;2020:1-7.

8. Haider MR, Brown MJ, Harrison S, Yang X, Ingram L, Bhochhibhoya A, et al. Sociodemographic factors affecting viral load suppression among people living with HIV in South Carolina. AIDS Care. 2021;33(3):290-8.

9. Sunkanmi F, Paul Y, Peter D, Nsikan A, Joseph J, Opada E, et al. Factors Influencing Viral Load Nonsuppression among People Living with HIV (PLHIV) in Borno State, Nigeria: A Case of Umaru Shehu Ultra-Modern Hospital. J Adv Med Med Res. 2020;32(3):98-105.

10. Dixon-Umo OT, Ikpeme EE. Viral suppression and predictors among adolescents receiving care for HIV/AIDS in a tertiary health centre in Uyo, South-South, Nigeria. J AIDS HIV Res. 2020;12(2):9-16.

11. Yiltok E, Agada C, Zoakah R, Malau A, Tanyishi D, Ejeliogu E, et al. Clinical profile and viral load suppression among HIV positive adolescents attending a tertiary hospital in North Central Nigeria. J Med Trop. 2020;22(2):133.

12. Federal Ministry of Health (FMOH). Nigeria Health Facility Registry. https://hfr.health.gov.ng/. Accessed 30 Sep 2021.

13. Raftery AE. Bayesian Model Selection in Social Research (with Discussion by Andrew Gelman \& Donald B. Rubin, and Robert M. Hauser, and a Rejoinder). 1994.

14. R Core Team. R: The R Project for Statistical Computing. 2021. https://www.r-project.org/. Accessed 30 Sep 2021.

15. Bulage L, Ssewanyana I, Nankabirwa V, Nsubuga F, Kihembo C, Pande G, et al. Factors Associated with Virological Non-suppression among HIV-Positive Patients on Antiretroviral Therapy in Uganda, August 2014-July 2015. BMC Infect Dis. 2017;17(1):326.

16. Kipp W, Alibhai A, Saunders LD, Senthilselvan A, Kaler A, Konde-Lule J, et al. Gender differences in antiretroviral treatment outcomes of HIV patients in rural Uganda. AIDS Care. 2010;22:271-8.

17. Boullé C, Kouanfack C, Laborde-Balen G, Boyer S, Aghokeng AF, Carrieri MP, et al. Gender differences in adherence and response to antiretroviral treatment in the Stratall Trial in rural district hospitals in Cameroon. JAIDS J Acquir Immune Defic Syndr. 2015;69(3):355-64.

18. Girum T, Wasie A, Lentiro K, Muktar E, Shumbej T, Difer M, et al. Gender disparity in epidemiological trend of HIV/AIDS infection and treatment in Ethiopia. Arch Public Health. 2018;76(1):51.

19. Desta AA, Woldearegay TW, Futwi N, Gebrehiwot GT, Gebru GG, Berhe AA, et al. HIV virological nonsuppression and factors associated with non-suppression among adolescents and adults on antiretroviral therapy in northern Ethiopia: a retrospective study. BMC Infect Dis. 2020;20(1):4.

20. Munyaneza F, Ntaganira J, Nyirazinyoye L, Birru E, Nisingizwe MP, Gupta N, et al. Community-based accompaniment and the impact of distance for HIV patients newly initiated on antiretroviral therapy: early outcomes and clinic visit adherence in rural Rwanda. AIDS Behav. 2018;22(1):77-85.

21. United States Center for Disease Control. Evidence of HIV Treatment and Viral Suppression in Preventing the Sexual Transmission of HIV. 2020. 
22. Diress G, Dagne S, Alemnew B, Adane S, Addisu A. Viral load suppression after enhanced adherence counseling and its predictors among high viral load HIV seropositive people in North Wollo Zone public hospitals, Northeast Ethiopia, 2019: retrospective cohort study. AIDS Res Treat. 2020;2020:19.

23. van Liere GAFS, Lilian R, Dunlop J, Tait C, Rees K, Mabitsi M, et al. High rate of loss to follow-up and virological non-suppression in HIV-infected children on antiretroviral therapy highlights the need to improve quality of care in South Africa. Epidemiol Infect. 2021;149:e88.

24. Kim MH, Wanless RS, Caviness C, Golin R, Amzel A, Ahmed S, et al. Multi-month prescription of antiretroviral therapy amongst children and adolescents: experiences from the Baylor International Pediatric AIDS initiative (BIPAI) in six African countries. J Acquir Immune Defic Syndr 1999. 2018;78(Suppl 2):S71-80.

25. Govindasamy D, Meghij J, Negussi EK, Baggaley RC, Ford N, Kranzer K. Interventions to improve or facilitate linkage to or retention in pre-ART (HIV) care and initiation of ART in low- and middle-income settings - a systematic review. J Int AIDS Soc. 2014;17(1):19032.

26. Ariyo O, Ozodiegwu ID, Doctor HV. The influence of the social and cultural environment on maternal mortality in Nigeria: Evidence from the 2013 demographic and health survey. PLOS ONE. 2017;12(12):e0190285.

27. Wang $\mathrm{C}, \mathrm{Cao} \mathrm{H}$. Persisting regional disparities in modern contraceptive use and unmet need for contraception among Nigerian women. BioMed Res Int. 2019;2019:1-9.

\section{Figures}

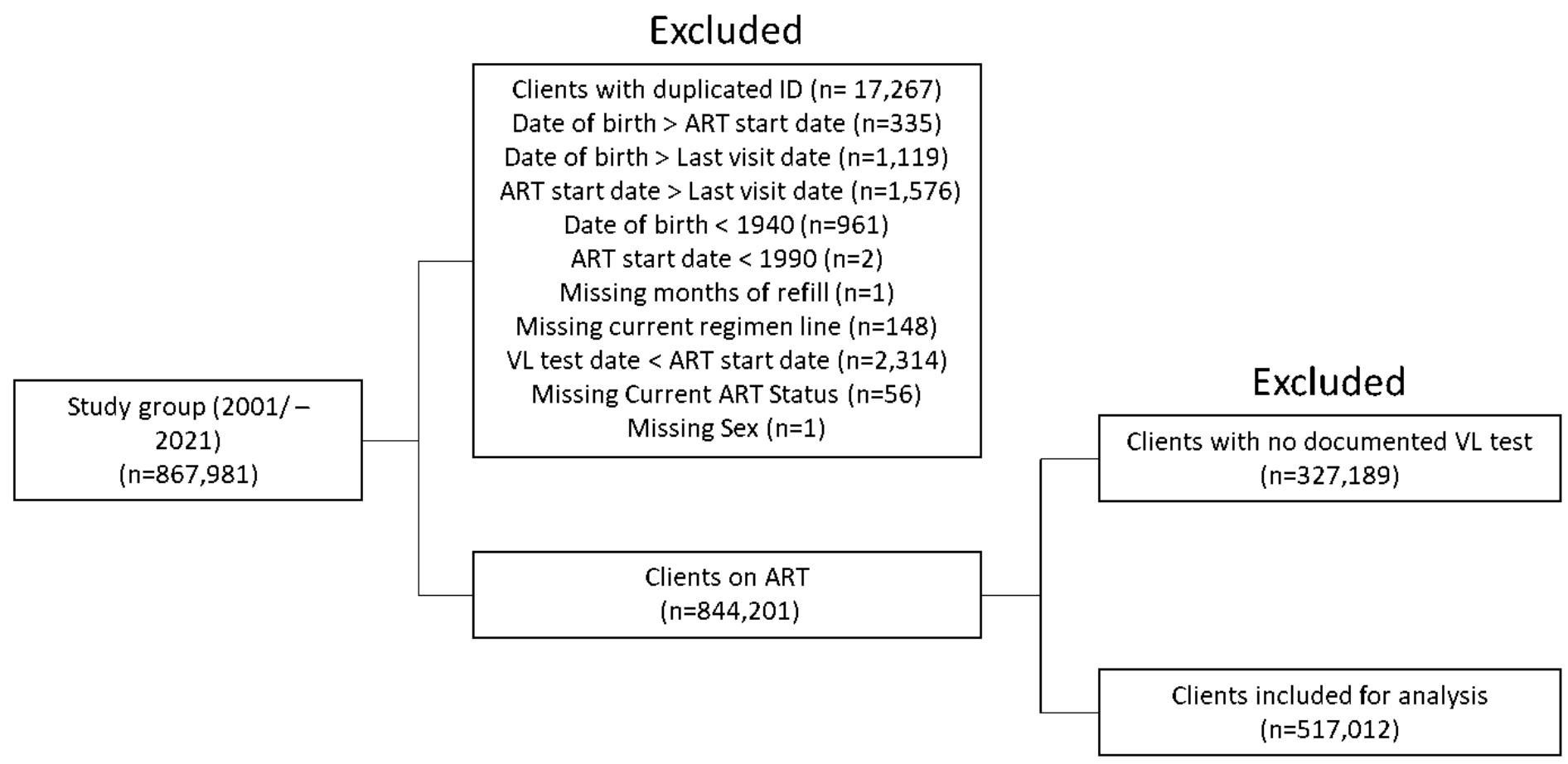


Figure 1

Data cleaning process, excluded data, and study population subset analyzed 